\title{
Lumières et révolutions en Amérique latine
}

Patrice Bret et Annie Duprat

\section{(2) OpenEdition}

\section{Journals}

Édition électronique

URL : https://journals.openedition.org/ahrf/12092

DOI : 10.4000/ahrf.12092

ISSN : 1952-403X

Éditeur :

Armand Colin, Société des études robespierristes

Édition imprimée

Date de publication : 1 septembre 2011

Pagination : 3-7

ISBN : 978-2-200-92700-4

ISSN : 0003-4436

\section{Référence électronique}

Patrice Bret et Annie Duprat, "Lumières et révolutions en Amérique latine », Annales historiques de la Révolution française [En ligne], 365 | Juillet-septembre 2011, mis en ligne le 01 septembre 2014, consulté le 22 avril 2022. URL : http://journals.openedition.org/ahrf/12092 ; DOI : https://doi.org/ 10.4000/ahrf.12092 


\section{LUMİ̀RES ET RÉVOLUTIONS EN AMÉRIQUE LATINE}

Patrice BRET

Annie DUPRAT

En proposant ce thème au comité de rédaction des Annales historiques de la Révolution française, nous avions le projet de réaliser en quelque sorte le pendant du numéro spécial précédent ( $n^{\circ} 363$, janviermars 2011), dirigé par Carla Hesse et Timothy Tackett, qui portait sur les relations entre la Révolution française et l'Amérique du Nord. Mais, plutôt que de pointer des différences stricto sensu géographiques, le numéro que nous présentons ici a choisi une approche mêlant, d'une part, les transferts culturels et scientifiques d'un continent à l'autre, et, d'autre part, les conditions de la formation et d'une effectivité de l'opinion publique.

Ces deux pôles thématiques permettent de mettre en évidence le rôle qu'y ont tenu les idées des Lumières dans le combat pour les indépendances et l'élaboration des textes politiques fondateurs des nouvelles républiques. Ils illustrent aussi la vitalité des recherches sur cet espace latino-américain des deux côtés de l'Atlantique. Revivifiée à l'occasion du bicentenaire de la Révolution française, une historiographie dynamique a montré l'importance des indépendances de l'Amérique latine, qui ont suivi celles des États-Unis et d'Haïti, dont la présence transparaît ici avec une acuité variable selon les articles.

Le choix a été également d'incarner ces approches en analysant quelques itinéraires individuels aussi différents que ceux de Juan Germán Roscio, théoricien républicain vénézuélien, d'Esteban Morel médecin français du Mexique victime de l'Inquisition, ou de José de Medina Galindo, gouverneur civil et militaire d'une province néogrenadine loyaliste. 
Retraçant le parcours et l'action du colonel Medina Galindo dans la vice-royauté de Grenade, Daniel Gutiérrez s'attarde sur les relations entre le gouverneur et les populations indiennes dans la province de Riohacha, située à quatre jours de navigation à peine de la Jamaïque. Depuis la guerre d'indépendance nord-américaine, les commerçants anglais, mais aussi, quoique dans une moindre mesure, hollandais, danois et français, ont réussi à entretenir des relations très étroites avec la nation guajira. On comprend que dans ces conditions le gouvernement de la vice-royauté pouvait être des plus délicats. Le colonel Medina Galindo, gouverneur de la province de Riohacha jusqu'à sa mort (1813), est contraint à louvoyer entre des intérêts divergents jusqu'à ce que le gouvernement de la province soit une nouvelle fois divisé entre une autorité militaire et une autorité politique. Au bout du compte, l'exemple de Medina Galindo démontre comment les patriciens néo-grenadins ont permis de préserver le fragile équilibre des petites communautés périphériques.

La culture des Lumières se développe au Portugal par l'intermédiaire des cafés, des salons de lecture, de la presse (la Gazette de Lisbonne) ou des sociétés savantes (voir la création en 1779 de l'Académie Royale des Sciences de Lisbonne). Les chocs successifs causés par la Révolution française, puis l'invasion de l'Espagne par les troupes de Napoléon $\mathrm{I}^{\mathrm{er}}$ et la difficile politique d'équilibre conduite par la couronne portugaise, écartelée entre son alliance avec l'Angleterre et la peur d'une victoire française en Espagne, finissent par contraindre la Cour de Lisbonne à embarquer à destination du Brésil. Guilherme Neves et Lúcia Bastos nous racontent ensuite comment, malgré une importante circulation de pamphlets et de périodiques aux idées libérales, la politique resta confinée à l'espace privé et la religion demeura la forme dominante de structuration du monde. L'indépendance du Brésil en 1822, conduite par l'héritier du trône lusitanien, s'est limitée à une dispute avec le Portugal pour l'hégémonie à l'intérieur de l'empire.

Les écrits des naturalistes brésiliens Alexandre Rodrigues Ferreira, Manuel Arruda da Câmara et José Bonifácio de Andrada e Silva auxquels est consacré l'article de Lorelaì Kury permettent de comprendre comment l'identité des nouvelles nations se construit aussi à travers des débats scientifiques autonomes. Comme partout en Europe au XVIII ${ }^{\mathrm{e}}$ siècle, les savants ne cessent de réfléchir aux rapports entre les hommes et la nature; au contraire de ce qu'écrivait Buffon, Ferreira, Câmara et José Bonifácio sont convaincus qu'il existe une spécificité brésilienne, en étroite relation avec son climat tropical et la luxuriance de sa végétation... 
L'espace andin, caractérisé par la présence nombreuse de populations d'origine indienne et par des révoltes antérieures dont le souvenir est encore vif, connait en 1794 une «affaire des placards » avec l'apparition de pamphlets à Santafé de Bogotá. On y trouve en particulier une traduction en espagnol de la Déclaration des Droits de l'Homme et du Citoyen proclamée en France en 1789. Se développe dès lors l'épisode de « la sourde rumeur », nommée parfois la rumeur d'un « second Paris », ce qui montre bien l'influence des idées de la Révolution française dans ces territoires américains bien éloignés. Le vice-roi José de Ezpeleta, qui avait combattu aux côtés des Insurgents avec Miranda, observe les agissements des publicistes Eugénio Espejo et Antonio de Nariño d'abord avec une certaine distance. Puis vient le temps de la répression, bien tardive pour sauver les intérêts de la couronne espagnole comme le montre Georges Lomné. La simultanéité de l'apparition de ces pasquins à Bogotá et à Mexico, en août 1794, ne manquera pas de surprendre. C'est dans ce contexte que le vice-roi Branciforte conduit également en NouvelleEspagne la répression dont Morel est victime l'année suivante. Mais l'analyse du dossier de l'Inquisition par Liliana Schifter, Patricia Aceves et Patrice Bret, témoigne aussi des résistances de la société à la diffusion des Lumières.

Deux articles sont consacrés au cas du Venezuela, celui de Clément Thibaud, qui examine l'action de Juan Germán Roscio, théoricien et promoteur du premier républicanisme vénézuélien. Roscio en fut même l'un des plus grands théoriciens latino-américains avec le Mexicain Servando Teresa de Mier et l'Équatorien Rocafuerte sur qui il eut une profonde influence comme le développe Clément Thibaud. Député et membre de l'exécutif confédéral, Roscio fait paraître en 1811 un Patriotisme de Nirgua et abus des rois. Exilé à Philadelphie, il publie encore en 1817 Le triomphe de la liberté sur le despotisme alors que la cause de l'indépendance paraissait perdue. Si l'évocation du parcours de Roscio ne permet pas de s'attaquer aux "causes» de l'indépendance en une sorte de téléologie rétrospective, son itinéraire éclaire plutôt les relations entre l'expérience sociopolitique d'un sujet de l'Empire et un destin révolutionnaire fasciné par la question républicaine. En d'autres termes, comment Roscio, ce notable respecté de Caracas, devint-il révolutionnaire? Pourquoi ce dévot $\mathrm{du}$ « pouvoir tyrannique des monarchies chrétiennes », comme il se décrit lui-même, finit-il par revêtir le bonnet phrygien? Ce thème en cache un autre, d'importance : la question des hiérarchies racialisées, qui sous-tend son choix d'un républicanisme militant. Pour sa part, Véronique Hébrard s'attache à montrer comment l'élite de Caracas, pensée comme le moteur 
de la révolution a développé des arguments pour organiser un Congrès, élu en octobre 1810, comme siège de la représentation. Les débats porteront non seulement sur les conditions d'une juste représentation politique des citoyens mais également sur les relations " centre/périphérie », loin d'être simples. Le concept d'opinion publique sert de toile de fond à ces enjeux liés à la représentation. Pour ce faire, deux débats qui se livrent au sein du Congrès sont utilisés par Véronique Hébrard : celui sur la division de la province de Caracas et celui sur l'éventuel transfert du siège du Congrès, deux éventualités motivées par la crainte de la tyrannie du centre et de son opinion.

Il n'était pas possible, dans le cadre étroit de ce numéro, de couvrir l'ensemble des colonies ibériques d'Amérique, ni tous les thèmes; d'autres espaces (Argentine, Chili, etc.) ont fait l'objet d'études récentes, publiées ou à paraître. Mais le dossier présenté rend néanmoins compte de la diversité de situations locales que l'on ne saurait réduire à un quelconque schéma simpliste de transfert d'idées. Il laisse aussi deviner la complexité de l'identité créole, fondatrice des nouveaux états, ellemême construite dans cette diversité. De multiples exemples auraient pu illustrer la complexité et les contradictions des échanges intellectuels et politiques avec l'Europe, tels ceux du prêtre José Antonio de Alzate y Ramírez et de l'afrancesado José María de Lanz, tous deux natifs du Mexique. Le premier, savant et journaliste mexicain, correspondant de l'Académie des sciences de Paris et du Jardin botanique de Madrid, s'efforce, durant trente années, d'introduire dans son pays les découvertes scientifiques et techniques de France et d'Europe, en fondant tour à tour quatre journaux savants, victimes de la censure vice-royale entre 1768 et 1795. Mais lorsque la monarchie espagnole envoie en NouvelleEspagne des savants et ingénieurs pour moderniser les institutions savantes, il oppose les savoirs locaux à la classification de Linné ou à la méthode du traitement du minerai d'argent de Born et à la nouvelle chimie de Lavoisier. Le second, jeune officier de la Marine espagnole, ne retourne jamais au Mexique : il choisit la République française en 1793, enseigne dans une école d'application de l'École polytechnique sous le Directoire puis dans la nouvelle école des travaux publics de Madrid (Escuela de Caminos y Canales); préfet et chef de bureau ministériel sous le roi Joseph, il passe à Londres au retour des Bourbons, sert en Argentine puis dans la Grande Colombie de Bolivar avant de mourir en exil en France.

Le présent numéro spécial de notre revue, s'il consacre des études fondatrices sur le rôle des Lumières en Amérique latine, n'est pas clos 
sur lui-même; il nous a fallu limiter le nombre des contributeurs. Aussi formulons-nous le souhait que d'autres chercheurs confient aux Annales historiques de la Révolution française les résultats de leurs travaux.

Patrice BRET Centre Alexandre Koyré Centre de recherche en histoire des sciences et des techniques (UMR 8560 du CNRS) patrice.bret@yahoo.fr

Annie Duprat CICC 95000 Cergy-Pontoise CNRS/ISCC/Laboratoire communication et politique annie.duprat@orange.fr 